\title{
Stress myocardial perfusion imaging in the elderly
}

\author{
Atif Alzahrani, MBBS, and Terrence D. Ruddy, MD (i) ${ }^{a}$ \\ ${ }^{a}$ Cardiology, University of Ottawa Heart Institute, Ottawa, ON, Canada \\ Received May 31, 2016; accepted May 31, 2016 \\ doi:10.1007/s12350-016-0585-1
}

\section{See related article, pp. 63-71}

\section{INCREASING ELDERLY POPULATION WITH SIGNIFICANT CORONARY ARTERY DISEASE (CAD)}

The elderly population is growing rapidly. By 2050, there will be over 25 million adults of age $\geq 80$ years alive in the United States. ${ }^{1}$ The mortality and morbidity of cardiovascular disease has increased disproportionately in the elderly age group. ${ }^{2}$ For example, individuals $\geq 75$ years of age experience over $60 \%$ of the burden of myocardial infarctions and represent only $6 \%$ of the population of the United States. ${ }^{2}$ The prevalence of CAD is greater in the elderly population and represents the most frequent cause of death in the aged. ${ }^{3}$ This increased prevalence of CAD in the elderly is due to age and greater prevalence of diabetes mellitus Type 2, dyslipidemia, and obesity.

\section{ROLE OF STRESS MYOCARDIAL PERFUSION IMAGING (MPI)}

The clinical diagnosis of CAD is more difficult in the elderly due to atypical or minimal symptoms on presentation. ${ }^{4}$ Older patients are less active than younger patients and have less typical exertional angina. Thus, stress MPI may be particularly useful in the elderly for the detection of $\mathrm{CAD}$, following progression, guiding management, and determining prognosis. However, the value of imaging in the elderly is less well defined, as the elderly subgroup have

Reprint requests: Terrence D. Ruddy, Cardiology, University of Ottawa Heart Institute, 40 Ruskin Street, Room H1220, Ottawa, ON K1Y 4W7, Canada; truddy@ottawaheart.ca

J Nucl Cardiol 2018;25:72-4.

$1071-3581 / \$ 34.00$

Copyright (c) 2016 American Society of Nuclear Cardiology. been usually not or under enrolled in most clinical trials. ${ }^{2}$ Since sensitivity and specificity are related to the population prevalence of CAD and older patients have a higher prevalence of CAD, sensitivity of MPI tends to be greater in the elderly with less specificity. The choice of stress agent for MPI may be more limited in the elderly. Exercise ability is often limited due to decreased aerobic capacity with age or other conditions such as deconditioning, peripheral vascular disease, arthritis, neuromuscular disease, or obesity. Thus, pharmacologic stress with vasodilators including dipyridamole, adenosine, adenosine triphosphate (ATP, not approved in the US), or regadenoson is used in the majority of the elderly unable to exercise or having left bundle branch block or paced rhythm. Much less frequently, dobutamine stress is used in patients unable to exercise and with significant bronchospasm, or sinus node disease or second- or third-degree atrioventricular block (without a functioning pacemaker). ${ }^{5}$

The elderly may have more logistical difficulties due to frailty with diminished reserves in strength and function, ${ }^{6}$ mobility issues and inability to lie flat for long acquisition times. This population may benefit from short acquisition times with usual radiation doses now possible with new gamma camera technology and software.

\section{PROGNOSTIC STUDIES WITH STRESS MPI IN THE ELDERLY}

Early studies have demonstrated the medium-term prognostic value of exercise MPI in elderly patients. Using exercise MPI, Steingart et $\mathrm{al}^{7}$ reported followup for a mean of 4.4 years in 626 patients of $\geq 65$ years of age and showed the independent predictive values of age, the extent of ischemia, exercise capacity, and male gender as a predictors of subsequent death or nonfatal infarction. However, the addition of extent of ischemia in the multivariate models made a small predictive contribution in addition to age, gender, and exercise capacity. Using exercise thallium MPI, Valeti et $\mathrm{al}^{8}$ reported followup of a mean duration of 6.4 years in 247 
patients of age $\geq 75$ years and demonstrated the additional predictive value of the MPI data for classification of patients into low- and high-risk groups. The annual cardiac mortality using summed stress scores was $0.8 \%$ for low-risk and $5.8 \%$ for high-risk patients.

Later studies have demonstrated the short- and medium-term prognostic value of exercise and/or vasodilator MPI in the elderly. Using gated MPI with Tc-99m tetrofosmin, DeWinter et $\mathrm{al}^{9}$ reported followup for a median of 25.9 months in 294 patients of age $\geq 75$ years undergoing exercise $(n=103)$, exercise and dipyridamole $(n=48)$, or dipyridamole stress $(n=$ 143). The summed rest score was a significant independent predictor of all-cause mortality and cardiac death. LV functional data provided independent and incremental information above clinical and perfusion MPI data for prediction of all-cause death (resting left ventricular ejection fraction) and cardiac death (left ventricular endsystolic volume). Using gated MPI with Tc-99m sestamibi, Nagao et al $^{10}$ reported a mean followup of 3.4 years in 175 patients of age $\geq 75$ years evaluated with exercise $(n=49)$ or vasodilator stress $(n=126)$ and demonstrated that stress-induced myocardial ischemia (summed difference score) was the only independent predictor for subsequent cardiac events using multivariate analysis. Using dual-isotope MPI, Hachamovitch et $\mathrm{al}^{11}$ described followup of 5200 patients $\geq 75$ years of age undergoing exercise $(n=2158,41 \%)$ or adenosine stress $(n=3042,59 \%)$ with mean followup of 2.8 years and a subset with extended followup of a mean of 6.2 years. Multivariate analysis showed the incremental predictive values of ischemia and fixed defects over clinical information for prediction of cardiac death and further enhancement of risk stratification with ejection fraction data. An interaction between early treatment and ischemia was present such that Ischemia was associated with increasing survival with early revascularization as compared to medical therapy having improved outcomes in the setting of little or no ischemia. Using ATP nongated MPI with Tc-99m-MIBI, Yao et $\mathrm{al}^{12}$ described mean followup data of 3.45 years in 415 patients and demonstrated the predictive value for summed stress score $>8$ for cardiac events in patients of age $\geq 70$ years.

One study using dobutamine stress by Schinkel et $\mathrm{al}^{13}$ demonstrated the prognostic value of Tc-99m Tetrofosmin nongated MPI in the elderly with followup of a mean of 3.3 years in 272 patients $\geq 65$ years of age. Multivariate analysis showed the incremental predictive values of a fixed or reversible defect and of the summed stress score over clinical data for prediction of total mortality or cardiac death.

In this issue of the journal, Roest et al describe the very long-term 14-year prognostic value of dobutamine stress Tc-99m tetrofosmin MPI in 272 consecutive patients $\geq 65$ years of age and unable to perform exercise testing. ${ }^{14}$ The mean 3.3-year followup for this population has been previously reported. ${ }^{13}$ At the time of patient enrollment between 1995 and 1999, dobutamine was the preferred pharmacologic stress in this nuclear cardiology laboratory for patients unable to exercise. Elsewhere, many of these patients would have been candidates for pharmacologic stress using vasodilator agents. Followup was complete for 270 (99.3\%) patients and 23 patients undergoing coronary artery revascularization less than 60 days following testing were excluded. Imaging used a 2-day protocol. Images were analyzed for the presence of fixed and/or reversible perfusion defects and a summed stress score was calculated as an estimate of the extent and severity of perfusion defects. Followup endpoints included allcause mortality, cardiac mortality, and nonfatal myocardial infarction. During median followup of 14 years, 168 $(68 \%)$ of the patients died (all-cause mortality) and 56 (23\%) died due to cardiac causes. Nonfatal MI occurred in $19(8 \%)$ patients. Cumulative survival curves were calculated using the Kaplan-Meier method, with comparison of the survival curves using the log rank test. The event-free survival for all-cause mortality for patients with an abnormal MPI was significantly worse than in patients with a normal MPI. Of note, the annual all-cause mortality was high in patients with normal scans $(5.1 \%$ per year at 5 years and $4.6 \%$ per year at 10 years of followup) and greater in the patients with the abnormal scans $(9.1 \%$ per year at 5 years and $6.1 \%$ per year at 10 years of followup). The annual all-cause event rate for patients with normal scans was much higher than the usual low event rate expected in patients with normal scans and consistent with the known worse prognosis of patients unable to perform an exercise test. ${ }^{15}$ The difference in all-cause mortality event rates between normal and abnormal scans became smaller over time probably reflecting noncardiac causes of death. The Kaplan-Meier survival curves for cardiac mortality with and without nonfatal myocardial infarction showed a lower risk in patients with a normal MPI with the event rate difference maintained over time. Univariable and multivariable Cox proportional hazard regression model was used to determine the incremental value of MPI parameters over clinical variables for prediction of cardiac events. An abnormal scan and a reversible defect predicted all-cause mortality. An abnormal MPI, reversible defect, and summed stress score (SSS) predicted cardiac mortality and hard cardiac events. Thus, dobutamine stress Tc-99m tetrofosmin MPI provided incremental prognostic value for the very long-term prediction of cardiovascular events in elderly patients unable to perform exercise testing. 


\section{NEED FOR STRESS MPI FOR SELECTION OF ELDERLY FOR REVASCULARIZATION}

The use of revascularization is increasing in the elderly. Registry studies have shown an increase in the number of elderly undergoing emergency and nonemergency percutaneous coronary intervention and more cardiovascular events at 30-day followup in the elderly. ${ }^{16,17}$ As well, results with coronary bypass surgery in the elderly ( $>80$ years) have improved with lower perioperative risks than previously reported and longterm survival similar to an age-adjusted population. ${ }^{18}$ However, revascularization has significant risks and costs which need to be justified by patient outcomes. Selection of elderly patients for aggressive intervention vs optimized medical therapy is best justified by prognostic data which can be provided by stress MPI. Prospective multicenter studies are needed to assess the cost-effectiveness of MPI-guided decision making for management of the elderly with suspected or known CAD.

\section{References}

1. Pecer G. US Bureau of the Census: Projections of the population of the United States by age, sex, and race: 1988 to 2080. Current Population Reports, Series P-25, No. 1018. Washington, DC: US Government Printing Office; 1989.

2. Alexander KP, Newby LK, Cannon CP, Armstrong PW, Gibler WB, Rich MW, et al. Acute coronary care in the elderly, Part 1. Circulation 2007;115:2549-69.

3. Odden MC, Coxson PG, Moran A, Lightwood JM, Goldman L, Bibbins-Domingo K. The impact of the aging population on coronary artery disease in the United States. Am J Med 2011;124:827-33.

4. Rogers WJ, Canto JG, Lambrew CT, Tiefenbrunn AJ, Kinkaid B, Shoultz DA, et al. Temporal trends in the treatment of over 1.5 million patients with myocardial infarction in the US from 1990 to 1999: The National Registry of Myocardial Infarction 1,2 and 3. J Am Coll Cardiol 2000;121:2056-63.

5. Henzlova M, Duvall WL, Einstein AJ, Travin MI, Verberne HJ. ASNC imaging guidelines for SPECT, nuclear cardiology procedures: Stress, protocols and tracers. J Nucl Cardiol 2016;23:60639.

6. Fried LP, Tangen CM, Walston J, Newman AB, Hirsch C, Gottdiener J, et al. Frailty in older adults: Evidence for a phenotype. J Gerontol A Biol Sci Med Sci 2001;56(3):M146-56.

7. Steingart RM, Hodnett P, Musso J, Feuerman M. Exercise myocardial perfusion imaging in elderly patients. J Nucl Cardiol 2002;9:573-80.
8. Valeti US, Miller TD, Hodge DO, Gibbons RJ. Exercise singlephoton emission computed tomography provides effectives risk stratification of elderly men and elderly women. Circulation 2005;111:1771-6.

9. De Winter O, Velghe A, Van de Veire N, De Bondt P, De Buyzere $\mathrm{M}$, Van De Wiele C, et al. Incremental prognostic value of combined perfusion and function assessment during myocardial gated SPECT in patients aged 75 years or older. J Nucl Cardiol 2005; 12:662-70.

10. Nagao T, Chikamori T, Hida S, Igarashi Y, Kuwabara Y, Nishimura $\mathrm{S}$, et al. Quantitative gated single-photon emission computed tomography with $99 \mathrm{mTc}$ sestamibi predicts major cardiac events in elderly patients with known or suspected coronary artery disease: The QGS-prognostic value in the elderly (Q-PROVE) study. Circ J 2007;71:1029-34.

11. Hachamovitch R, Kang X, Amanullah AM, Abidov A, Hayes SW, Friedman JD, et al. Prognostic implications of myocardial perfusion single-photon emission computed tomography in the elderly. Circulation 2009;120:2197-206.

12. Yao Z, Zhu H, Li W, Chen C, Wang H, Shi L, et al. Adenosine triphosphate stress myocardial perfusion imaging for risk stratification of patients aged 70 years and older with suspected coronary artery disease. J Nucl Cardiol 2016. doi:10.1007/s12350015-0355-5.

13. Schinkel AF, Elhendy A, Biagini E, van Domburg RT, Valkema $\mathrm{R}$, Rizello V, et al. Prognostic stratification using dobutamine stress 99m-Tc-tetrofosmin myocardial perfusion SPECT in elderly patients unable to perform exercise testing. J Nucl Med 2005;46: 12-8.

14. Roest S, Boiten HJ, van Domburg RT, Valkema R, Schinkel AFL. Prediction of 14-year cardiovascular outcomes by dobutamine stress ${ }^{99 \mathrm{~m}} \mathrm{Tc}$-tetrofosmin myocardial perfusion SPECT in elderly patients unable to perform exercise testing. J Nucl Cardiol 2016. doi:10.1007/s12350-016-0516-1.

15. Johnson NP, Schimmel DR Jr, Dyer SP, Leonard SM, Holly TA. Survival by stress modality in patients with a normal myocardial perfusion study. Am J Cardiol 2011;107:986-9.

16. Johnman C, Oldroyd KG, MacKay DF, Slack R, Pell AC, Flapan $\mathrm{AD}$, et al. Percutaneous intervention in the elderly. Changes in case-mix and periprocedural outcomes in 31758 patients treated between 2000 and 2007. Circ Cardiovasc Interv 2010;3:341-5.

17. Antonsen L, Jensen LO, Thayssen P, Christiansen EH, Junker A, Tilsted HH, et al. Comparison of outcomes of patients $\geq 80$ years of age having percutaneous coronary intervention according to presentation (stable vs unstable angina pectoris/non-ST-segment elevation myocardial infarction vs ST-segment elevation myocardial infarction). Am J Cardiol 2011;108:1395-400.

18. Saxena A, Dinh DT, Cheng-Hon Y, Reid CM, Billah B, Smith JA, et al. Critical analysis of early and late outcomes after isolated coronary artery bypass surgery in elderly patients. Ann Thorac Surg 2011;92:1703-11. 